\title{
Eigenvalue-Based RF Interference Detector for Multi-Antenna Wireless Communications
}

\author{
Tilahun M. Getu, Member, IEEE, Wessam Ajib, Senior Member, IEEE, \\ and René Jr. Landry, Member, IEEE
}

\begin{abstract}
Radio frequency interference (RFI) is occurring in both satellite and terrestrial communication systems. In order to mitigate RFI efficiently, it has to be detected robustly. Toward this end, through the computation of an eigenvalue-based test statistic, an eigenvalue-based blind RFI detector is proposed for single-input multiple-output systems that may suffer from RFI. For medium to large interference-tonoise ratio (INR) regimes and under sample starved settings, Monte-Carlo simulations corroborate that the proposed blind detector manifests a comparable detection performance with a generalized likelihood ratio test (GLRT) detector fed with the knowledge of the signal of interest (SOI) channel, and a matched subspace detector fed with the knowledge of the SOI and RFI channels. Such performance signifies the applicability of the proposed RFI detector for real-time applications.
\end{abstract}

\section{Index Terms}

RFI detection and excision, eigenvalue detector, GLRT detector, matched subspace detector.

T. M. Getu was with the École de Technologie Supérieure (ÉTS), Montréal, QC H3C 1K3, Canada, and also with the Université du Québec à Montréal (UQAM), Montréal, QC H2L 2C4, Canada (e-mail: tilahun-melkamu.getu.1@ens.etsmtl.ca). He is the corresponding author.

W. Ajib is with the Université du Québec à Montréal (UQAM), Montréal, QC H2L 2C4, Canada (e-mail: ajib.wessam@uqam.ca).

R. Jr. Landry is with the École de Technologie Supérieure (ÉTS), Montréal, QC H3C 1K3, Canada (e-mail: renejr.landry@etsmtl.ca).

Parts of this paper were presented at the IEEE Vehicular Technology Conference (VTC2018-Fall) held in Chicago, IL, USA as of Aug. 2018. The authors have no competing interests. 


\section{INTRODUCTION}

\section{A. Related Works}

Due to out-of-band emissions by nearby transmitters and harmonics, jammers, spoofers, and meaconers, radio frequency interference (RFI) is being increasingly common in microwave radiometry [1], radio astronomy (RA) [2], and satellite communications (SatCom) [3], [4]. Regarding SatCom, 93\% of the industrial applications suffer from interference-as reported in [5]- and RFI is a potential threat to global navigation satellite system [6]. RFI also happens in cognitive radio systems for imperfect spectrum sensing [7] (as also analytically implicated through [8]); ultra-wideband communications due to the prevalent narrowband interferers [9]; and radar because of the inevitable jammers [10]. As such a widely occurring RFI must be first detected so as to be excised efficiently, researchers have paid attention, throughout the years, to the research field of RFI detection.

In the aforementioned regard, the state-of-the-art encompasses considerable number of RFI detectors. Mentioning the main ones, the RFI detector in asynchronous pulse blanking [11], kurtosis detector (KD) [12], fast Fourier transforms-based RFI detector [13], a precorrelationbased RFI detector [14], and transformed-domain detectors [15]. In general, these RFI detectors deployed frameworks that did not lead to analytical performance characterizations, which are often missing. On the other hand, the performance characterization of a given RFI detector is not a straightforward undertaking, as the distribution and parameters of the impinging RFI are generally unknown. Such a lack of knowledge makes the existing hypothesis testing frameworks [16], [17] hardly useful with regard to the aforementioned undertaking. Highlighting the latest research advancements pertaining to this research field, meanwhile, a power-based broadband RFI detector and an energy-based RFI detector are investigated in [18] and [19], respectively. However, these RFI detectors rely on the knowledge of the noise power and they are detectors proposed, mainly, for single-antenna systems. Thus, it is of an academic and practical significance to develop robust multi-antenna RFI detectors whose performance characterizations shall also be pursued.

Mathematically, the multi-antenna RFI detection problem can be related to the adaptive radar detection problem — considered in $[20]-[23]$ - by exchanging the underlying RFI and the signal of interest (SOI) while presuming a receiving reference antenna. For the adaptive signal detection in homogeneous Gaussian disturbance and structured interference, [21] has derived several 
theoretically founded detectors which are proved to be the function of the maximal invariant statistic (MIS) corroborating their constant false alarm rate (CFAR) property. Following [10], [22] exploits the principle of invariance to surmount the problem of adaptive vector subspace signal detection in a partially homogeneous Gaussian disturbance plus structured interference. In particular, [22] derives an MIS which is shown to coincide with the adaptive normalized matched filter [24] (adaptive coherence estimator [25]) in a complementary subspace of the structured interference. Thereafter, several well-known test statistics are derived and shown to be statistically equivalent to the MIS. Similarly, [23] deals with the adaptive detection of point-like targets in a possibly heterogeneous environment. In a mathematical sense, some of the electronic countercountermeasures $(\mathrm{ECCM})$ techniques $[26]-[28]$ are also related to the problem of multi-antenna RFI detection.

\section{B. Motivation}

Despite the mathematical resemblance, the detection techniques of [20]-[23] cannot be adopted as robust multi-antenna RFI detection techniques. The presumption of known left and right subspaces for the signal and interference makes the unifying framework of [21] hardly practical for multi-antenna RFI detection. Similarly, the assumptions that a target signature and a structured interference belong to known subspaces make [22] unattractive for multi-antenna RFI detection. Because of the assumption regarding a known subspace spanned by the interference steering vectors, [23] is also unattractive. Meanwhile, adapting the techniques of [29] devised for a mismatched signal model is either challenging or complex, as the RFI target vectors are generally unknown and time-variant. Furthermore, as the impinging RFI may not be Gaussian and its distribution is generally unknown, adapting the ECCM related techniques—such as [26], [27], and [28, Ch. 12]—as robust multi-antenna RFI detection techniques would be hardly realistic.

Because it requires identifying the type of RFI which could be narrowband, broadband, continuous wave, or pulsed RFI [3], [6], the development of a robust multi-antenna RFI detector is challenging. In this regard, an RFI detector should robustly detect any kind of RFI unlike KD which fails to detect Gaussian (near Gaussian) RFI(s) [12]. To be attractive for real-time applications, an RFI detector should not also rely on a large number of samples. Moreover, an RFI detector shall also be able to detect very weak RFI, as several such RFIs can make the communication (system) unreliable, especially in SatCom and RA which manifest a received signal whose strength is usually under the noise floor [2]. 
In another regard, eigenvalue-based detectors $[30]-[32]$ have been proposed for spectrum sensing in the context of cognitive radios. These detectors exhibit an attractive detection performance and their blindness makes them practically appealing. They do not also rely on the power spectral density of the noise unlike conventional energy detectors [33]-[37]. Meanwhile, eigenvalues in the Karhunen-Loève transform domain were deployed to detect RFI, as detailed in [38]. Nonetheless, time-domain eigenvalue detection had not been investigated until recently. In line with this specific motivation, [39] has disseminated a preliminary study regarding an eigenvaluebased multi-antenna RFI detection. Following this lead, we make the following contributions.

\section{Contributions}

Based on the lead of [39], this article presents a full-fledged investigation on eigenvalue-based RFI detector and its performance assessment. Being consequences of the conducted investigation, the contributions of this paper are itemized beneath.

- For single-input multiple-output (SIMO) systems that may suffer from RFI, an eigenvaluebased RFI detector is proposed and studied in detail.

- The performance of the investigated RFI detector is assessed extensively via Monte-Carlo simulations.

\section{Organization}

Section II describes the considered system model. Section III presents the problem formulation and the proposed detection. Section $\mathrm{IV}$ reports the simulation results. Finally, Section $\mathrm{V}$ presents the concluding remarks and research outlook of this paper.

\section{E. Notation}

Italic letters, lower-case boldface letters, and upper-case boldface letters denote scalars, vectors, and matrices, respectively. $\mathbb{C}^{N_{R}}, \mathbb{C}^{N \times M}$, and $\mathbb{H}^{N_{R} \times N_{R}}$ are the sets of $N_{R}$-dimensional vectors of complex numbers, of $N \times M$ complex matrices, and of $N_{R} \times N_{R}$ Hermitian matrices, respectively. $\sim, \mid, \triangleq,(\cdot)^{T}$, and $(\cdot)^{H}$ denote distributed as, conditioned on (under), equal by definition, transpose, and Hermitian, respectively. $\in, \boldsymbol{A}(:, i), \boldsymbol{A}(:, i: j)$, and $\operatorname{diag}(\cdot)$ stand for element of (belongs to), the $i$-th column of $\boldsymbol{A}$, the columns of $\boldsymbol{A}$ between its $i$-th and $j$-th columns including its $i$-th and $j$-th columns, and (block) diagonal matrices, respectively. $\|\cdot\|$, $\boldsymbol{I}_{N_{R}}$, and $\mathbf{0}_{N \times M}$ denote Euclidean norm, an $N_{R} \times N_{R}$ identity matrix, and an $N \times M$ zero matrix, 


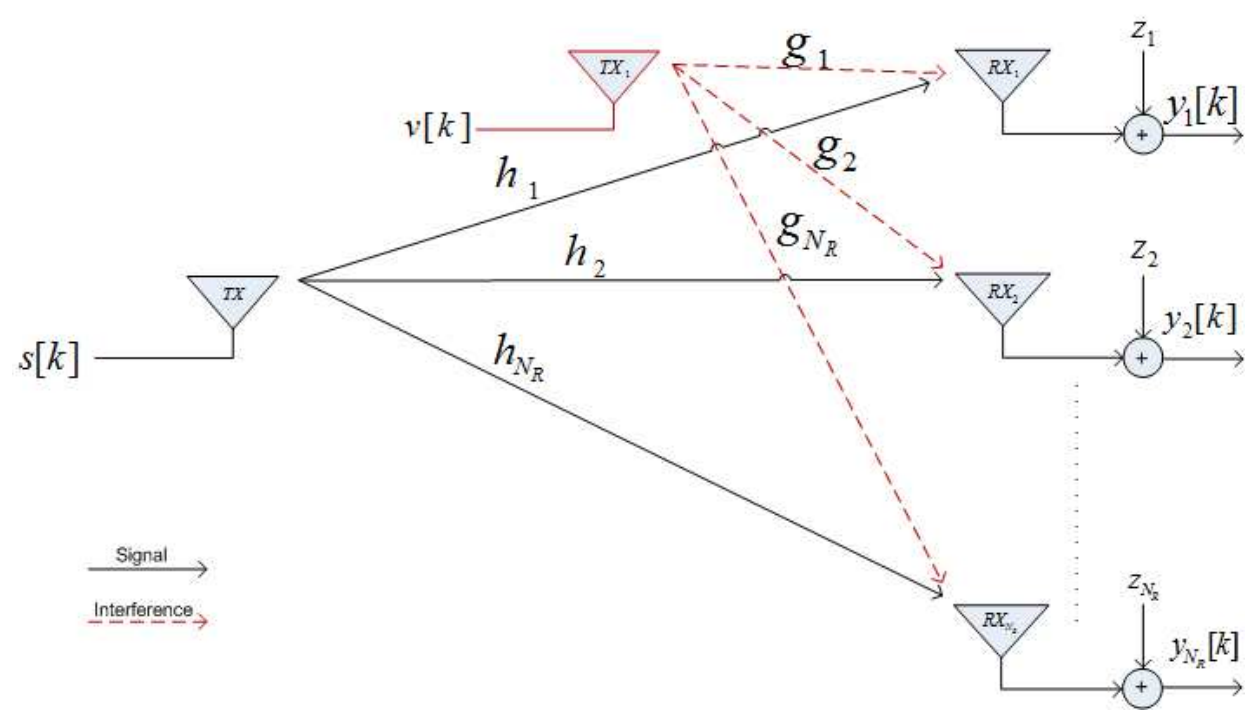

Fig. 1. A baseband schematic of the considered SIMO system suffering from an RFI.

respectively. $\mathcal{N}\left(\mathbf{0}, \sigma^{2} \boldsymbol{I}_{N_{R}}\right)$, and $\mathcal{C N}\left(\mathbf{0}, \boldsymbol{I}_{N_{R}}\right)$ represent normal distribution with mean zero and covariance matrix $\sigma^{2} \boldsymbol{I}_{N_{R}}$ and circularly symmetric complex normal distribution with mean zero and covariance matrix $\sigma^{2} \boldsymbol{I}_{N_{R}}$, respectively.

\section{System Model}

First, we assume that the received passband signal is downconverted to its baseband equivalent and sampled at the Nyquist rate. In line with this assumption, we consider a SIMO system that may suffer from an RFI as depicted in Fig. 1. Along with the reception of the transmitted SOI, an RFI emitted by a nearby single-antenna source might also be received by the $N_{R}$ antennas. For this scenario, the received signal contaminated by noise and an impinging RFI is expressed as

$$
\boldsymbol{y}[k]=\boldsymbol{h} s[k]+\boldsymbol{g} v[k]+\boldsymbol{z}[k] \in \mathbb{C}^{N_{R}},
$$

where $\boldsymbol{y}[k]$ is the $k$-th sample of the received multi-antenna signal; $\boldsymbol{h}=\left[h_{1}, h_{2}, \ldots, h_{N_{R}}\right]^{T}$ $\in \mathbb{C}^{N_{R}}$ is the flat fading SOI channel gain vector assumed constant during the RFI detection interval; $s[k]$ is the $k$-th unknown and deterministic symbol of the SOI; $\boldsymbol{g}=\left[g_{1}, g_{2}, \ldots, g_{N_{R}}\right]^{T} \in$ $\mathbb{C}^{N_{R}}$ is the flat fading RFI channel gain vector assumed constant during the RFI detection interval; $v[k]$ is the $k$-th unknown and deterministic symbol of the RFI; and $\boldsymbol{z}[k] \sim \mathcal{C N}\left(\mathbf{0}, \sigma^{2} \boldsymbol{I}_{N_{R}}\right)$ 
is - with an unknown power of $\sigma^{2}$ - a zero mean circularly symmetric complex additive white Gaussian noise (AWGN) vector, which manifests a spatially uncorrelated noise.

Inferring from (1), the RFI-free received multi-antenna signal is equated as

$$
\boldsymbol{y}[k]=\boldsymbol{h} s[k]+\boldsymbol{z}[k] \in \mathbb{C}^{N_{R}} .
$$

Referring to the maximum number of sources_of our problem setting_-being two, we assume that $N_{R}>2$. Moreover, the remainder of this manuscript presumes that $\boldsymbol{h}, \boldsymbol{g}$, and $\boldsymbol{z}[k]$ are independent random variables.

\section{Problem Formulation And Proposed Detection}

\section{A. Problem Formulation}

A binary hypothesis test is formulated from (1) and (2) as

$$
\boldsymbol{y}[k]= \begin{cases}\boldsymbol{h} s[k]+\boldsymbol{g} v[k]+\boldsymbol{z}[k] & : H_{1} \\ \boldsymbol{h} s[k]+\boldsymbol{z}[k] & : H_{0},\end{cases}
$$

where $\left\{H_{0}, H_{1}\right\}$ are, respectively, hypotheses regarding the absence and presence of the RFI and $1 \leq k \leq N$ for $N$ being the number of intercepted per-antenna samples. Stacking the observation vectors of $N$ sampling intervals,

$$
\boldsymbol{y}= \begin{cases}\boldsymbol{H} \boldsymbol{s}+\boldsymbol{G} \boldsymbol{v}+\boldsymbol{z} & : H_{1} \\ \boldsymbol{H} \boldsymbol{s}+\boldsymbol{z} & : H_{0},\end{cases}
$$

where $\boldsymbol{y}=\left[\boldsymbol{y}^{T}[1], \boldsymbol{y}^{T}[2], \ldots, \boldsymbol{y}^{T}[N]\right]^{T} \in \mathbb{C}^{N_{R} N}$ is the stacked multi-antenna received signal samples, $\boldsymbol{H}=\operatorname{diag}(\boldsymbol{h}, \boldsymbol{h}, \ldots, \boldsymbol{h}) \in \mathbb{C}^{N_{R} N \times N}$ is the SOI channel matrix, $\boldsymbol{G}=\operatorname{diag}(\boldsymbol{g}, \boldsymbol{g}, \ldots, \boldsymbol{g}) \in$ $\mathbb{C}^{N_{R} N \times N}$ is the RFI channel matrix, $\boldsymbol{s}=[s[1], s[2], \ldots, s[N]]^{T} \in \mathbb{C}^{N}$ is the SOI vector, $\boldsymbol{v}=$ $[v[1], v[2], \ldots, v[N]]^{T} \in \mathbb{C}^{N}$ is the RFI vector, and $\boldsymbol{z}=\left[\boldsymbol{z}^{T}[1], \boldsymbol{z}^{T}[2], \ldots, \boldsymbol{z}^{T}[N]\right]^{T} \in \mathbb{C}^{N_{R} N} \sim$ $\mathcal{C N}\left(\mathbf{0}, \sigma^{2} \boldsymbol{I}_{N_{R} N}\right)$ is the stacked multi-antenna noise vector. If $\boldsymbol{H}$ and $\boldsymbol{G}$ were known in the matched subspace detection problem stated via [40, eq. (2.4)], (4) and [40, eq. (2.4)] would be equivalent problems for $\boldsymbol{S}=\boldsymbol{H}, \boldsymbol{\phi}=\boldsymbol{s}$; and $\mu=1, \boldsymbol{H}=\boldsymbol{G}$, and $\boldsymbol{\theta}=\boldsymbol{v}$ : $^{1}$

The problem formulated in (4) can also be related to the adaptive radar signal detection problems of [20]-[23]. However, adopting these techniques is challenging, since they rely on known subspace(s). The problem can also be posed as a source enumeration problem [41]-[43]:

\footnotetext{
${ }^{1}$ With regard to $|40|$, please note that $\mu \boldsymbol{H} \boldsymbol{\theta}$ and $\boldsymbol{S} \boldsymbol{\phi}$ denote an information-bearing signal and an interference, respectively.
} 
"two sources" versus "one source". Nonetheless, the technique of [41] cannot be adopted here, as $\{s[k], v[k]\}$ are not necessarily Gaussian random variables (RVs). Besides, the information criterion rules [42], [43] are computationally complex, as they rely on the minimization of highly non-linear functions made of several maximum-likelihood estimates [44]. For known $\boldsymbol{h}$ and Gaussian $\{s[k], v[k]\}$, it is worth mentioning that the problem can also be recast in terms of a unified generalized likelihood ratio test (GLRT) based spectrum sensing framework of [45].

\section{B. Proposed Detection}

At first, the proposed eigenvalue-based RFI detector computes the sample covariance matrix $(\mathrm{SCM})$ as

$$
\hat{\boldsymbol{R}}_{y y}=\frac{1}{N} \sum_{k=1}^{N} \boldsymbol{y}[k] \boldsymbol{y}^{H}[k]=\frac{1}{N} \boldsymbol{Y} \boldsymbol{Y}^{H} \in \mathbb{H}^{N_{R} \times N_{R}},
$$

where $\boldsymbol{Y}=[\boldsymbol{y}[1], \boldsymbol{y}[2], \ldots, \boldsymbol{y}[N]] \in \mathbb{C}^{N_{R} \times N}$. Hereinafter, we assume that $N>N_{R}$ to ensure that all eigenvalues of the SCM are positive with probability one. Computing the singular value decomposition (SVD) of (5),

$$
\hat{\boldsymbol{R}}_{y y}=\hat{\boldsymbol{U}} \hat{\boldsymbol{\Sigma}} \hat{\boldsymbol{V}}^{H}=\left[\hat{\boldsymbol{U}}_{1} \hat{\boldsymbol{U}}_{2: N_{R}}\right] \hat{\boldsymbol{\Sigma}} \hat{\boldsymbol{V}}^{H} \stackrel{(a)}{=} \hat{\boldsymbol{U}} \hat{\boldsymbol{\Sigma}} \hat{\boldsymbol{U}}^{H},
$$

where $\hat{\boldsymbol{U}}_{1}=\hat{\boldsymbol{U}}(:, 1), \hat{\boldsymbol{U}}_{2: N_{R}}=\hat{\boldsymbol{U}}\left(:, 2: N_{R}\right)$, and $\hat{\boldsymbol{\Sigma}}=\operatorname{diag}\left(\hat{\sigma}_{1}, \hat{\sigma}_{2}, \ldots, \hat{\sigma}_{N_{R}}\right)$ for $\left\{\hat{\sigma}_{i}\right\}_{i=1}^{N_{R}}$ being the singular values - in a decreasing order-of the SCM, and $(a)$ emanates from the fact that an SCM is both a positive semi-definite and Hermitian matrix.

It shall be recalled that $\left\{\hat{\sigma}_{i}\right\}_{i=2}^{N_{R}}$ and $\left\{\hat{\sigma}_{i}\right\}_{i=3}^{N_{R}}$ are the noise eigenvalues under $H_{0}$ and $H_{1}$, respectively. Under $H_{1}$, if the interference-to-noise ratio (INR) is greater than the signal-tonoise ratio $(\mathrm{SNR}), \hat{\sigma}_{2}$ is contributed by the signal and the noise; whereas $\hat{\sigma}_{2}$ is contributed by the interference and the noise provided that the SNR is greater than the INR. Employing this intuition, the test statistic — that can flag the impinging RFI-is defined as

$$
T \triangleq \frac{\hat{\sigma}_{2}}{\sum_{i=3}^{N_{R}} \hat{\sigma}_{i}}
$$

For $\lambda$ being the decision threshold often set as per the desired false alarm rate (FAR), a decision rule follows as

$$
T \underset{H_{0}}{\stackrel{H_{1}}{\gtrless}} \lambda .
$$

Remark 1. Once the trace of the SCM is obtained, (7) can be computed via the first two dominant eigenvalues obtained efficiently using the power method [46, Chs. 7 and 8]. 


\begin{tabular}{|l|c|}
\hline Parameters & Assigned Value \\
\hline$N_{R}$ & 5 \\
\hline$\gamma_{s n r}$ & $0 \mathrm{~dB}$ \\
\hline$P_{s}$ & $10 \mathrm{~W}$ \\
\hline No. of realizations & $10^{5}$ \\
\hline
\end{tabular}

TABLE I

SiMULATION PARAMETERS IF NOT EXPLICITLY MENTIONED.

\section{Simulation Results}

Evaluated for a SIMO system, this section reports the simulation results regarding the performance of the proposed RFI detector, matched subspace detector (MSD) [40], and multiantenna detectors [47]-[49]. Unless otherwise mentioned, the conducted simulations employ the parameters of Table I]. Without loss of generality and similar to [50], [51], the independently distributed complex channel gains pertaining to the SOI and RFI—unless stated differently-are modeled by a Rayleigh fading, i.e., $\boldsymbol{h} \sim \mathcal{C N}\left(\mathbf{0}, \boldsymbol{I}_{N_{R}}\right)$ and $\boldsymbol{g} \sim \mathcal{C N}\left(\mathbf{0}, \boldsymbol{I}_{N_{R}}\right)$. Unless otherwise mentioned and without loss of generality, we consider a quadrature phase shift keying (QPSK) modulated SOI and RFI, i.e, $s[k]=\sqrt{P_{s} / 2}\left[s_{k}^{I}+j s_{k}^{Q}\right]$ and $v[k]=\sqrt{P_{v} / 2}\left[v_{k}^{I}+j v_{k}^{Q}\right]$ for $\left\{s_{k}^{I}, s_{k}^{Q}\right\} \in\{-1,1\} \times\{-1,1\}$ and $\left\{v_{k}^{I}, v_{k}^{Q}\right\} \in\{-1,1\} \times\{-1,1\}$.

The subsequent Monte-Carlo simulations of the proposed detector deploy the test statistic given by (7) and the decision rule expressed in (8). These simulations consider that the SNR and INR are, respectively, defined as $\gamma_{s n r}=\frac{\|\boldsymbol{H} \boldsymbol{s}\|^{2}}{N N_{R} \sigma^{2}}$ and $\gamma_{i n r}=\frac{\|\boldsymbol{G} \boldsymbol{v}\|^{2}}{N N_{R} \sigma^{2}}$. Having adjusted the power of the received SOI and the received RFI per these definitions of SNR and INR, the MonteCarlo simulations regarding the exhibited $P_{d}$ - by any considered detector-assume the reception of $H_{1}$ per a realization and average over the number of assumed realizations. Along with the simulation of $P_{d}$, the probability of miss-detection $\left(P_{m}\right)$ exhibited by any considered detector is simulated as $P_{m}=1-P_{d}$. On the other hand, the simulations concerning the FAR-manifested by any detector-assume a per-realization reception of $H_{0}$, whose SOI component is adjusted as per the SNR definition provided above, and average over the number of presumed realizations. Meanwhile, fixed for the detection performance assessment of the considered detectors, the decision thresholds rendering the desired FARs are obtained via Monte-Carlo simulations that average over $10^{6}$ independent realizations under $H_{0}$. Regarding the proposed eigenvalue-based 
RFI detector, hereinafter, performance comparison with MSD; performance comparison with multi-antenna detectors; and assessment of the manifested FAR and complementary receiver operating characteristics (CROC) are reported.

To imply that the $y$-axes of the subsequent plots are scaled using logarithmic scale, we labeled them as $\log P_{d}, \log P_{m}$, or $\log P_{f}$.

\section{A. Performance Comparison with MSD}

To compare the proposed detector and an MSD [40] which assumes real-valued signals, we emulate the transmission of a binary phase shift keying (BPSK) modulated SOI and BPSK modulated RFI over real-valued Rayleigh fading channels, i.e., $\boldsymbol{h} \sim \mathcal{N}\left(\mathbf{0}, \boldsymbol{I}_{N_{R}}\right)$ and $\boldsymbol{g} \sim \mathcal{N}\left(\mathbf{0}, \boldsymbol{I}_{N_{R}}\right)$. With respect to the desired FAR of 0.01 , we detect the presence of RFI using the proposed detector and the MSD derived for a subspace signal detection in subspace interference and noise of unknown level [40, Sec. VIII]. For a given realization, the performance of MSD is assessed via the $P_{D}$ expression given by [40, eq. (8.10)] and its respective FAR threshold is obtained via the $P_{F A}$ expression, also, equated in [40, eq. (8.10)]. As the NCP [40, eq. (8.7)] that comprises the closed-form detection expression [40, eq. (8.10)] varies for every realization, we average the per-realization detection performance of MSD over $10^{5}$ realizations. Similarly, the detection performance of the proposed RFI detector is assessed through Monte-Carlo simulations that also average over $10^{5}$ realizations. Whereas for the respective $P_{m}$ simulations, averaging over $10^{6}$ realizations is considered.

With respect to the aforementioned simulation settings, Figs. 2 and 3 , respectively, depict the $P_{d}$ and $P_{m}$ exhibited by the proposed RFI detector and MSD. As seen in Fig. 2 for $\gamma_{i n r} \geq 5$ $\mathrm{dB}$, the proposed RFI detector has a comparable detection performance with MSD fed with the knowledge of $\boldsymbol{H}$ and $\boldsymbol{G}$ though the proposed detector is a blind one. Concerning Fig. 3, even though it is outperformed-in the low INR regimes-by MSD fed with the knowledge of $\boldsymbol{H}$ and $G$, the proposed blind detector also enjoys a considerably small $P_{m}$, especially in the high INR regimes, manifested even for a sample starved setting as small as $N=50$.

\section{B. Performance Comparison with Multi-Antenna Detectors}

We compare the performance of the proposed detector with multi-antenna detectors [47][49] proposed for spectrum sensing in the context of cognitive radios [52]. To simulate the RFI detection performance of these detectors [47]-[49], we first assume the availability of the 


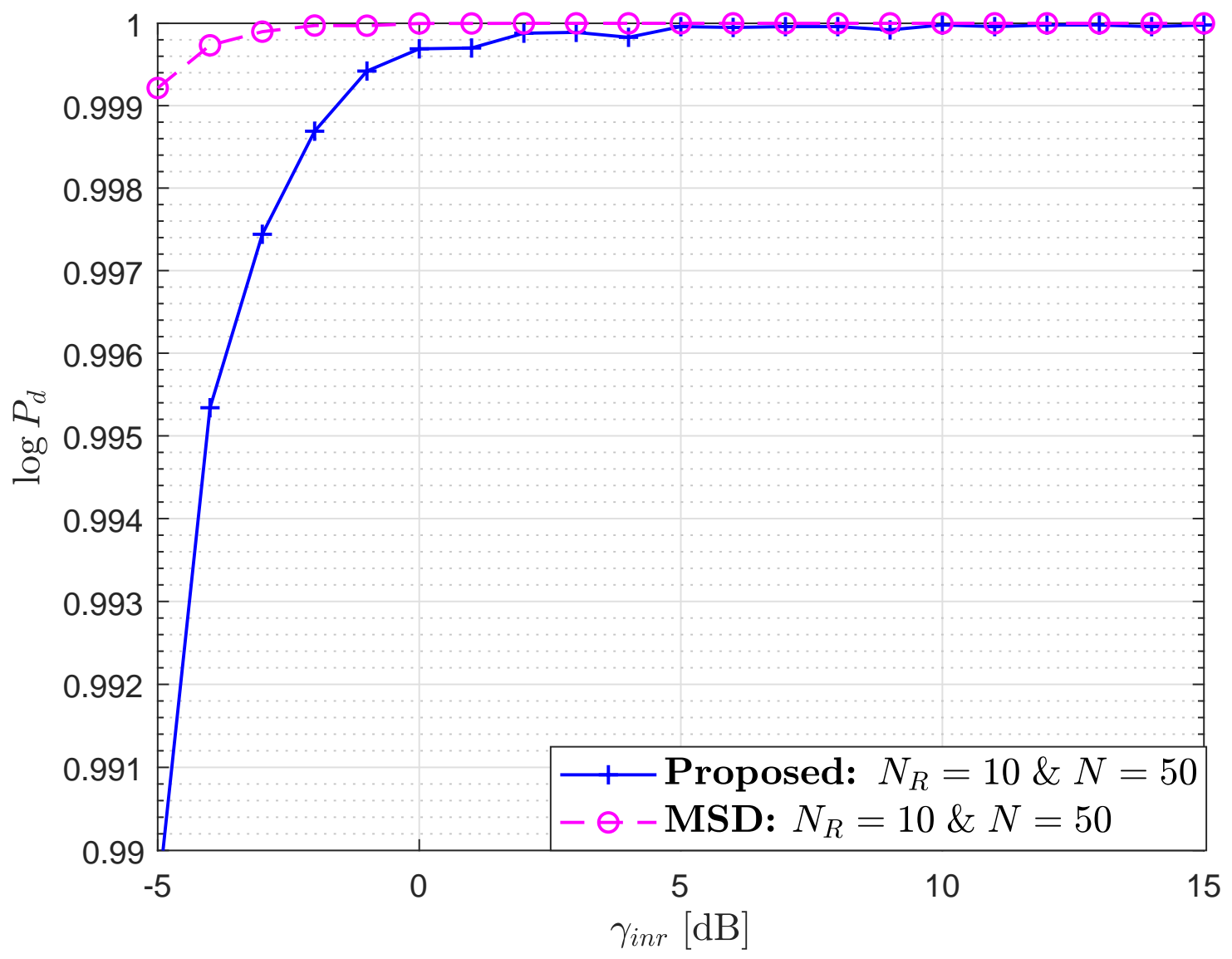

Fig. 2. $P_{d}$ versus $\gamma_{i n r}: P_{f}=0.01$.

knowledge of $\boldsymbol{h}$ and execute projection orthogonal to the SOI subspace using a projection matrix $\boldsymbol{P}=\boldsymbol{I}_{N_{R}}-\boldsymbol{h}\left(\boldsymbol{h}^{H} \boldsymbol{h}\right)^{-1} \boldsymbol{h}^{H}$. Thereafter, along with the proposed blind detector, we simulate the $F$-test based detector [47, eqs. (4) and (5)], blind GLRT [48, eq. (39)], multi-channel energy detection (MCED) [49, eq. (2)], and multi-channel energy detection with noise uncertainty (MCED-U) [49, eq. (3)].

To simulate the $F$-test based detector [47], the test statistic in [47, eq. (5)] is computed via a projection matrix $\boldsymbol{P}^{\prime}=\boldsymbol{I}_{N_{R}}-\boldsymbol{g}\left(\boldsymbol{g}^{H} \boldsymbol{g}\right)^{-1} \boldsymbol{g}^{H}$, by also assuming the knowledge of $\boldsymbol{g}$, after projecting orthogonal to the SOI using $\boldsymbol{P}$. Having projected orthogonal to the SOI subspace, blind GLRT is simulated via [48, eq. (39)]. Note that such a GLRT statistic was also reported in [49, eq. (13)]. After also projecting orthogonal to the SOI subspace, MCED and MCED$\mathrm{U}$ are, respectively, simulated via [49, eq. (2)] and [49, eq. (3)]. To simulate the detection 


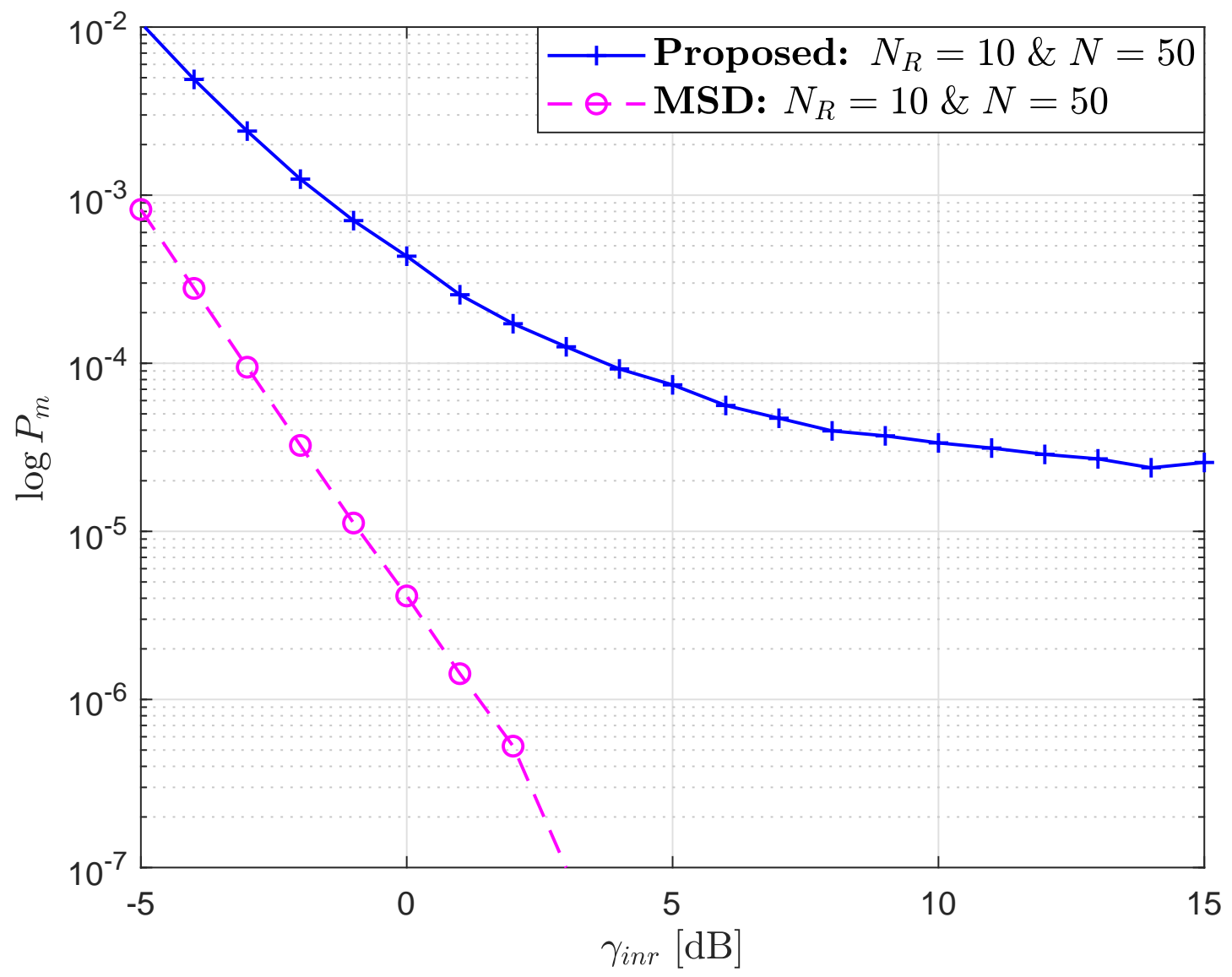

Fig. 3. $P_{m}$ versus $\gamma_{i n r}: P_{f}=0.01$ and $10^{7}$ channel realizations.

performance of MCED-U, we employ a constant noise uncertainty factor $\xi_{n u}=10 \log _{10} \eta_{n u}$ which is valid when the observation time is short [49]. Following the lead of [53], we assume that MCED-U overestimates $\sigma^{2}$ by a factor of uncertainty denoted by $\eta_{n u}$, i.e., $\hat{\sigma}^{2}=\eta_{n u} \sigma^{2}$, and compute its respective threshold rendering the considered desired FAR. Nevertheless, the detection performance of MCED-U is simulated using data with the exact noise variance $\sigma^{2}$.

Observing Fig. 4, the proposed blind RFI detector has a comparable detection performance with a GLRT fed with a perfect estimate of the SOI channel for the medium to high INR regimes. For the small INR regimes, the proposed detector outperforms the $F$-test detector [47, eqs. (4) and (5)], MCED [49, eq. (2)], and MCED-U [49, eq. (3)] with $\xi_{n u}=1 \mathrm{~dB}$ though the latter detectors are fed with the knowledge of the SOI channel. Such a performance manifested for a sample starved setting-as few as $N=50$-implicates the applicability of the proposed blind 
detector for a real-time detection of weak RFI(s) which usually occurs in SatCom and RA.

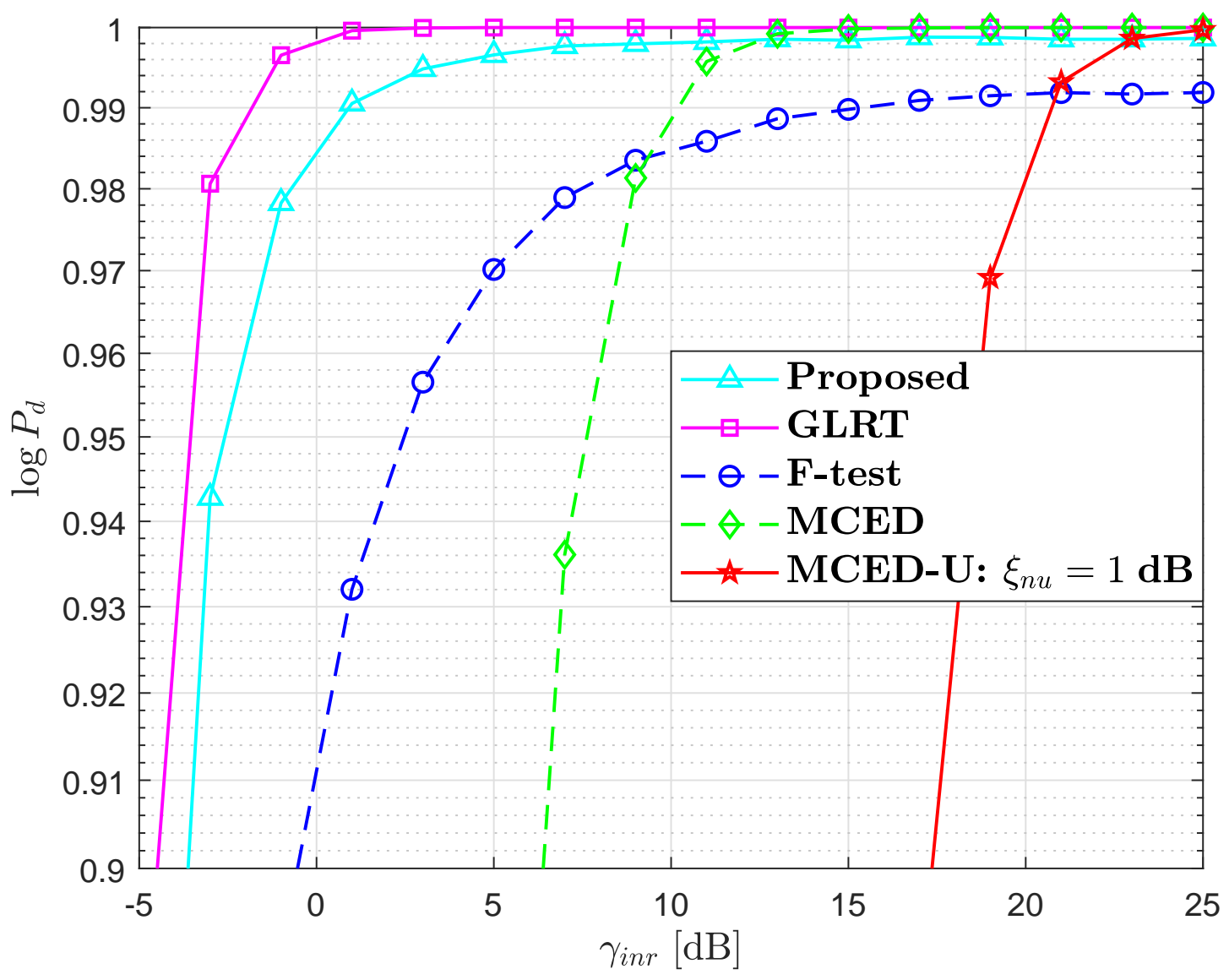

Fig. 4. $P_{d}$ versus $\gamma_{i n r}: N=50$ and $P_{f}=0.01$.

In order to offer further insight, we simulate the $P_{m}$ versus $\gamma_{i n r}$ performance curves as depicted in Fig. 5. For the small INR regimes, the proposed blind detector manifests a comparable missdetection performance with GLRT fed with the knowledge of the SOI channel. As the strength of the received RFI increases, MCED and MCED-U fed with both the knowledge of the SOI channel and the noise power-as ED requires the knowledge of the noise power-outperforms the proposed RFI detector. It is visible in Fig. 5 that the performance gains of MCED and MCED$\mathrm{U}$ are evident with a significantly small $P_{m}$ for a given INR. Summarizing the observations, however, from practicality and real-time processing perspectives, the proposed blind detector is attractive as manifested through its detection and miss-detection performance for both the small and the high INR regimes. 


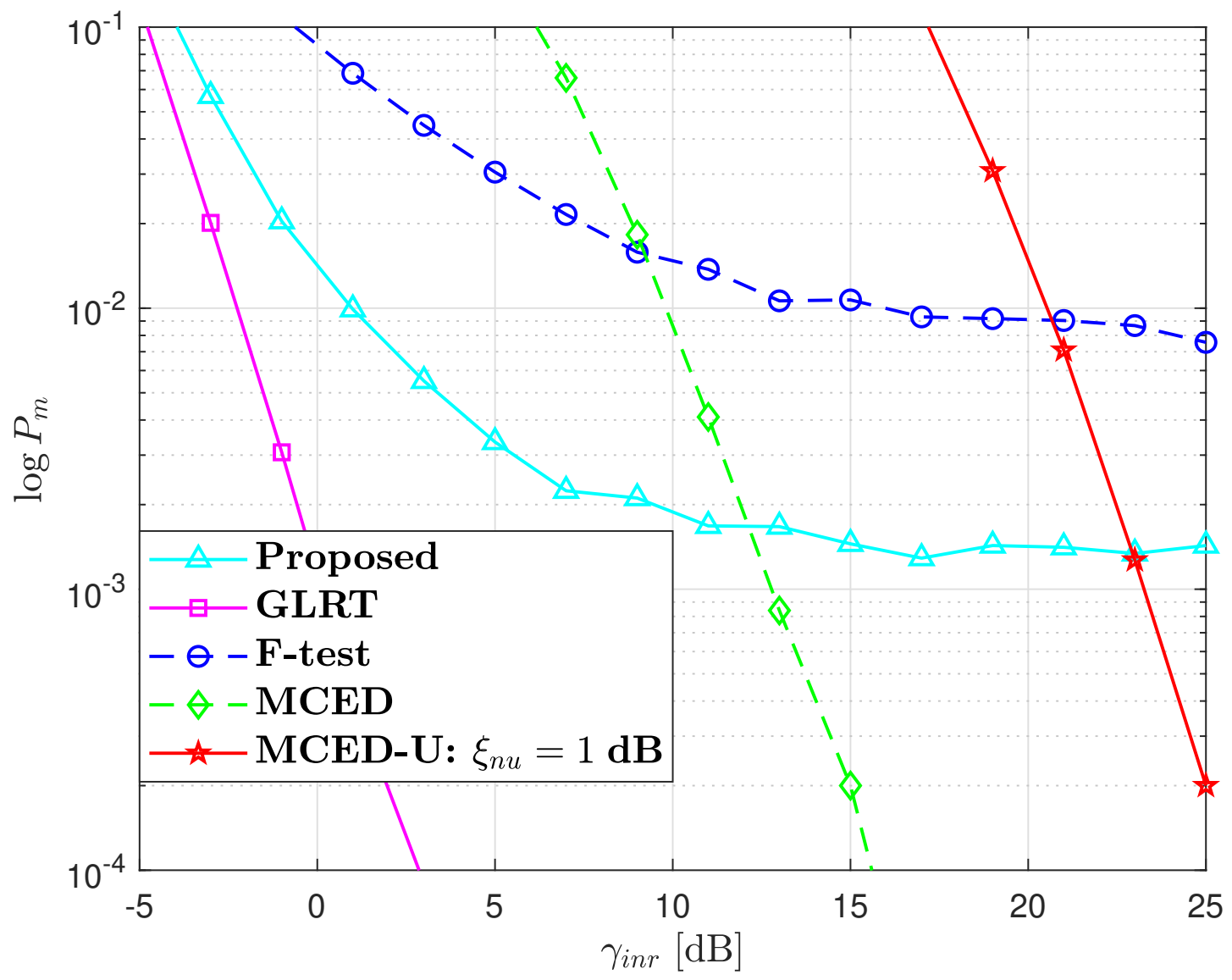

Fig. 5. $P_{m}$ versus $\gamma_{i n r}: N=50$ and $P_{f}=0.01$.

\section{Assessment of the Manifested FAR and CROC}

The impact of the number of received signal samples on the manifested FAR is assessed through Fig. 6. As depicted, the probability of false alarm becomes infinitesimally small as $N$ gets larger. It is also demonstrated that the CFAR constraint is not satisfied in the finite length regime if the threshold is set using asymptotic false alarm probability-like GLRT [48], [49]. As originally addressed by [54], the proposed detector also exhibits difficulties to satisfy the FAR constraint exactly like complex detectors.

At last, the simulation assessments are culminated by the assessment of the CROC exhibited by the proposed RFI detector. In order to depict the underlying CROC, the $P_{m}$ versus $P_{f}$ curves are depicted for different $N_{R}$ and $\gamma_{i n r}$ that comprise Fig. 7. As corroborated via Fig. 7, since the increment in INR or $N_{R}$ provides an improvement in an RFI detection and hence a smaller 


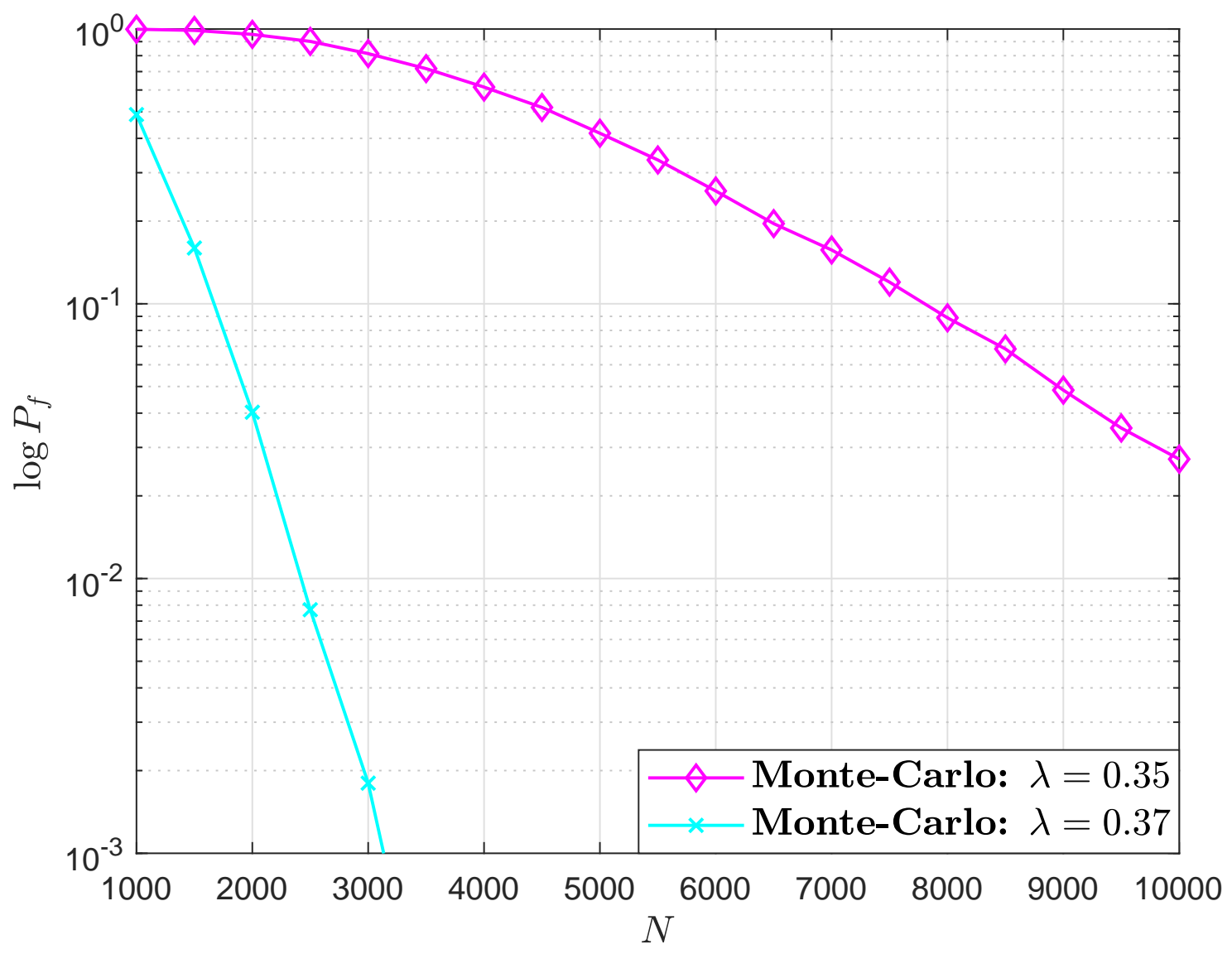

Fig. 6. Impact of $N$ on $P_{f}: 10^{4}$ realizations.

likelihood of missing the impinging RFI, the CROC curves shift inward with respect to $\gamma_{i n r}$ and $N_{R}$. In addition, Fig. 7 demonstrates the natural trade-off between $P_{m}$ and $P_{f}$.

\section{Concluding Remarks and Research Outlook}

\section{A. Concluding Remarks}

An eigenvalue-based blind RFI detector is proposed and studied for SIMO systems that may suffer from RFI. For sample starved settings and medium to large INR regimes, the conducted simulations corroborate that the proposed detector exhibits a comparable detection performance with a GLRT detector fed with the knowledge of the SOI channel, and an MSD fed with the knowledge of the SOI and RFI channels. Such performance reveals the attractiveness of the proposed RFI detector for real-time applications. 


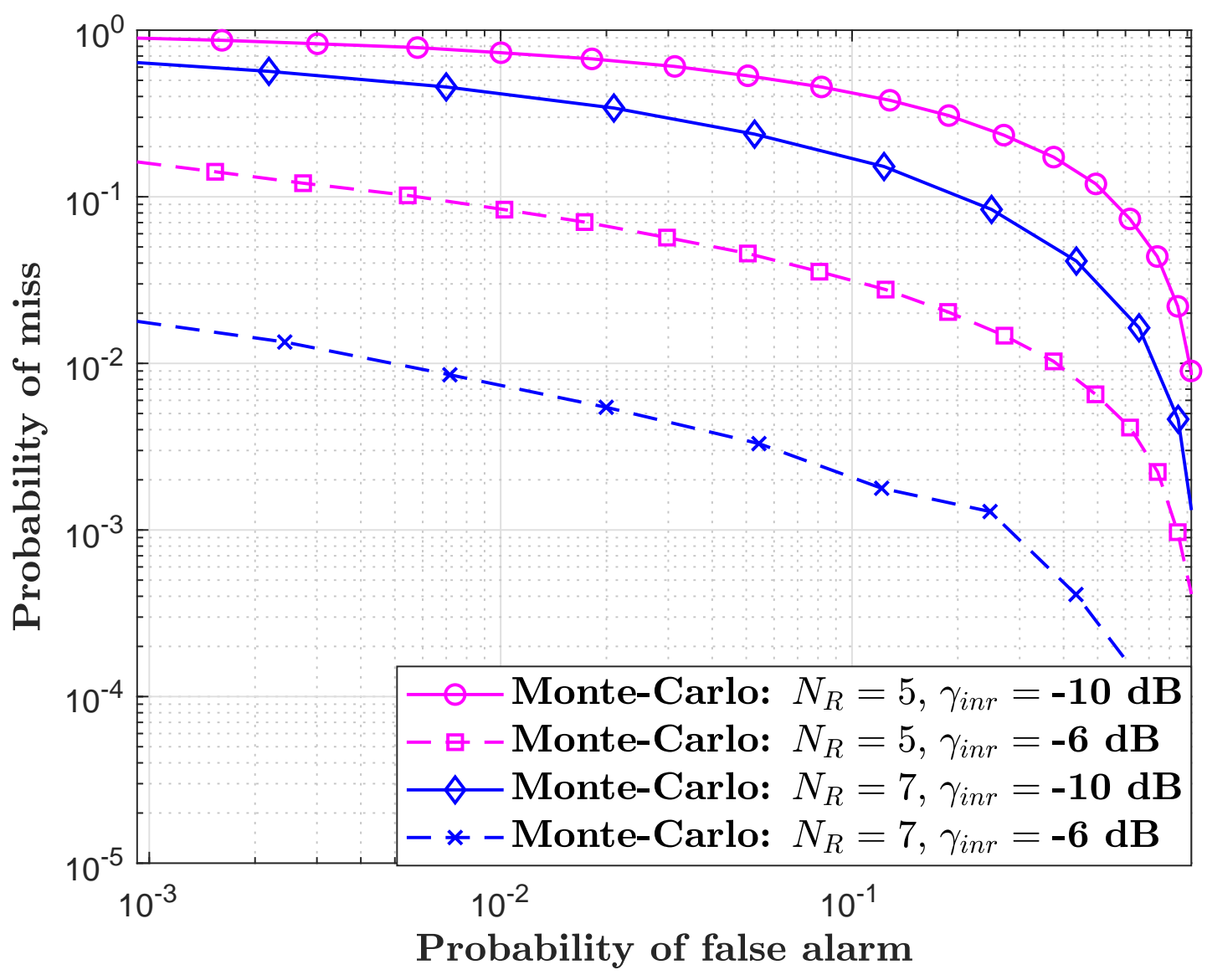

Fig. 7. Complementary ROC: $N=100$.

\section{B. Research Outlook}

If the values of $\boldsymbol{h}$ were known and $\{s[k], v[k]\}$ were Gaussian RVs, the underlying RFI detection problem could also be reformulated as the detection of rank-1 signals, which is a special case of the hypothesis test in [55, eq. (3)]. With respect to [55] which considers the detection of rank- $R(R \geq 1)$ signals with uncalibrated multiple antennas, the proposed eigenvalue detector can also be extended to the detection of rank- $R(R>1) \operatorname{RFI}(\mathrm{s})$. To practically realize such an extension, the channel order of the SOI and the RFI(s) are required and hence source enumeration techniques [41], [43], [56] would be, preliminarily, needed. In addition to the aforementioned extension, extension of the proposed RFI detector to the multiple-input multiple-output (MIMO) and massive MIMO systems [57], [58] — that might also consider a rank- $R(R>1)$ RFI(s) is worth addressing. Accounting for the inevitable calibration uncertainties of the $N_{R}$ antenna 
frontends similar to [53], [55], [59], consideration of independent and non-identically distributed (i.ni.d.) noises is also worth investigating for SIMO, MIMO, and massive MIMO systems [57], [58].

The exact performance analysis of the proposed eigenvalue-based RFI detector requires the probability distribution function (PDF) and cumulative distribution function (CDF) of (7). Unfortunately, deriving the CDF and PDF of (7) is mathematically intractable. While announcing this open problem, we encourage the interested reader to take courage so as to come up with the exact performance analysis of the proposed eigenvalue-based RFI detector by seizing these advancements [30], [60]-[66] regarding the PDFs and CDFs of different eigenvalue-based distributions. Eventually, having witnessed the emerging wide-applicability of high-dimensional probability and statistics [67], [68], future works shall devise a detailed exposition on the performance bounds of the proposed RFI detector.

\section{ACKNOWLEDGMENTS}

The authors acknowledge the funding provided by AVIO-601 Project which was sponsored by the Natural Sciences and Engineering Research Council of Canada (NSERC). They also acknowledge different anonymous reviewers whose objective criticisms and comments have helped them to improve the previous versions of this manuscript. 


\section{REFERENCES}

[1] B. Guner, J. T. Johnson, and N. Niamsuwan, "Time and frequency blanking for radio-frequency interference mitigation in microwave radiometry," IEEE Trans. Geosci. Remote Sens., vol. 45, no. 11, pp. 3672-3679, Nov. 2007.

[2] S. van der Tol and A. J. van der Veen, "Performance analysis of spatial filtering of RF interference in radio astronomy," IEEE Trans. Signal Process., vol. 53, no. 3, pp. 896-910, Mar. 2005.

[3] T. M. Nguyen, "Radio interference modeling and prediction for satellite operation applications," Air Force Research Laboratory and The Catholic Univ. of America, Tech. Rep., Aug. 2015.

[4] T. M. Getu, W. Ajib, and O. A. Yeste-Ojeda, "Tensor-based efficient multi-interferer RFI excision algorithms for SIMO systems," IEEE Trans. Commun., vol. 65, no. 7, pp. 3037-3052, Jul. 2017.

[5] Newtec and IRG, "93\% of the industry suffers from satellite interference," Sep. 2013. [Online]. Available: http://www.newtec.eu/article/release/93-of-the-industry-suffers-from-satellite-interference

[6] M. Wildemeersch and J. Fortuny-Guasch, "Radio frequency interference impact assessment on Global Navigation Satellite Systems,” EC Joint Research Centre, Security Tech. Assessment Unit, Tech. Rep., Jan. 2010.

[7] T. M. Getu, W. Ajib, and O. A. Yeste-Ojeda, "Efficient semi-blind channel estimators for SIMO systems suffering from broadband RFI," in Proc. IEEE Int. Conf. on Ubiquitous Wireless Broadband, Montreal, QC, Canada, Oct. 2015.

[8] A. A. A. Boulogeorgos, N. D. Chatzidiamantis, and G. K. Karagiannidis, "Spectrum sensing with multiple primary users over fading channels," IEEE Commun. Lett., vol. 20, no. 7, pp. 1457-1460, Jul. 2016.

[9] K. Shi, Y. Zhou, B. Kelleci, T. W. Fischer, E. Serpedin, and A. 1. Karsilayan, "Impacts of narrowband interference on OFDM-UWB receivers: Analysis and mitigation,” IEEE Trans. Signal Process., vol. 55, no. 3, pp. 1118-1128, Mar. 2007.

[10] A. De Maio and D. Orlando, "Adaptive radar detection of a subspace signal embedded in subspace structured plus Gaussian interference via invariance," IEEE Trans. Signal Process., vol. 64, no. 8, pp. 2156-2167, Apr. 2016.

[11] J. T. Johnson and S. W. Ellingson, "Examination of a simple pulse blanking technique for RFI mitigation," Radio Sci., vol. 40, no. 5, p. RS5S03, Jun. 2005.

[12] S. Misra, P. N. Mohammed, B. Guner, C. S. Ruf, J. R. Piepmeier, and J. T. Johnson, "Microwave radiometer radiofrequency interference detection algorithms: A comparative study," IEEE Trans. Geosci. Remote Sens., vol. 47, no. 11, pp. 3742-3754, Nov. 2009.

[13] A. T. Balaei and A. G. Dempster, "A statistical inference technique for GPS interference detection," IEEE Trans. Aerosp. Electron. Syst., vol. 45, no. 4, pp. 1499-1511, Oct. 2009.

[14] D. Borio, L. Camoriano, S. Savasta, and L. L. Presti, “Time-frequency excision for GNSS applications," IEEE Syst. J., vol. 2, no. 1, pp. 27-37, Mar. 2008.

[15] F. Dovis, L. Musumeci, N. Linty, and M. Pini, "Recent trends in interference mitigation and spoofing detection," Int. J. of Embedded and Real-Time Commun. Syst., vol. 3, no. 3, pp. 1-17, Jul./Sep. 2012.

[16] S. M. Kay, Fundamentals of Statistical Signal Processing: Detection Theory, Vol. II. Prentice-Hall, Englewood Cliffs, NJ, 1998.

[17] L. L. Scharf, Statistical Signal Processing: Detection, Estimation, and Time Series Analysis. Reading, MA, USA: AddisonWesley, 1991.

[18] T. M. Getu, W. Ajib, and R. Jr. Landry, "Power-based broadband RF interference detector for wireless communication systems," IEEE Wireless Commun. Lett., vol. 7, no. 6, pp. 1002-1005, Dec. 2018.

[19] T. M. Getu, W. Ajib, and R. Jr. Landry, "Performance analysis of energy-based RFI detector," IEEE Trans. Wireless Commun., vol. 17, no. 10, pp. 6601-6616, Oct. 2018. 
[20] D. Ciuonzo, A. De Maio, and D. Orlando, "A unifying framework for adaptive radar detection in homogeneous plus structured interference-Part I: On the maximal invariant statistic," IEEE Trans. Signal Process., vol. 64, no. 11, pp. 2894-2906, Jun. 2016.

[21] D. Ciuonzo and A. De Maio, and D. Orlando, "A unifying framework for adaptive radar detection in homogeneous plus structured interference-Part II: Detectors design,” IEEE Trans. Signal Process., vol. 64, no. 11, pp. 2907-2919, Jun. 2016.

[22] D. Ciuonzo, A. De Maio, and D. Orlando, "On the statistical invariance for adaptive radar detection in partially homogeneous disturbance plus structured interference,” IEEE Trans. Signal Process., vol. 65, no. 5, pp. 1222-1234, Mar. 2017.

[23] A. Aubry, A. De Maio, D. Orlando, and M. Piezzo, "Adaptive detection of point-like targets in the presence of homogeneous clutter and subspace interference," IEEE Signal Process. Lett., vol. 21, no. 7, pp. 848-852, Jul. 2014.

[24] E. Conte, M. Lops, and G. Ricci, “Adaptive matched filter detection in spherically invariant noise," IEEE Signal Process. Lett., vol. 3, no. 8, pp. 248-250, Aug. 1996.

[25] L. L. Scharf and L. T. McWhorter, "Adaptive matched subspace detectors and adaptive coherence estimators," in Proc. 30th Asilomar Conf. Signals, Syst., Comput., Nov. 1996, pp. 1114-1117.

[26] D. Orlando, "A novel noise jamming detection algorithm for radar applications," IEEE Signal Process. Lett., vol. 24, no. 2, pp. 206-210, Feb. 2017.

[27] F. Bandiera, A. Farina, D. Orlando, and G. Ricci, "Detection algorithms to discriminate between radar targets and ECM signals," IEEE Trans. Signal Process., vol. 58, no. 12, pp. 5984-5993, Dec. 2010.

[28] W. L. Melvin and J. A. Scheer, Eds., Principles of Modern Radar: Advanced Techniques. Edison, NJ, USA: SciTech, 2013.

[29] F. Bandiera and D. Orlando, "Advanced radar detection schemes under mismatched signal models," Synthesis Lectures Signal Process., vol. 4, no. 1, pp. 1-105, 2009.

[30] A. Kortun, M. Sellathurai, T. Ratnarajah, and C. Zhong, "Distribution of the ratio of the largest eigenvalue to the trace of complex Wishart matrices," IEEE Trans. Signal Process., vol. 60, no. 10, pp. 5527-5532, Oct. 2012.

[31] Y. Zeng and Y.-C. Liang, "Eigenvalue-based spectrum sensing algorithms for cognitive radio," IEEE Trans. Commun., vol. 57, no. 6, pp. 1784-1793, Jun. 2009.

[32] P. Bianchi, M. Debbah, M. Maida, and J. Najim, "Performance of statistical tests for single-source detection using random matrix theory," IEEE Trans. Inf. Theory, vol. 57, no. 4, pp. 2400-2419, Apr. 2011.

[33] F. F. Digham, M. S. Alouini, and M. K. Simon, "On the energy detection of unknown signals over fading channels," IEEE Trans. Commun., vol. 55, no. 1, pp. 21-24, Jan. 2007.

[34] A. A. D’Amico, U. Mengali, and E. A. de Reyna, "Energy-detection UWB receivers with multiple energy measurements," IEEE Trans. Wireless Commun., vol. 6, no. 7, pp. 2652-2659, Jul. 2007.

[35] S. P. Herath, N. Rajatheva, and C. Tellambura, "Energy detection of unknown signals in fading and diversity reception," IEEE Trans. Commun., vol. 59, no. 9, pp. 2443-2453, Sep. 2011.

[36] A. A. A. Boulogeorgos, N. D. Chatzidiamantis, and G. K. Karagiannidis, "Energy detection spectrum sensing under RF imperfections," IEEE Trans. Commun., vol. 64, no. 7, pp. 2754-2766, Jul. 2016.

[37] P. C. Sofotasios, E. Rebeiz, L. Zhang, T. A. Tsiftsis, D. Cabric, and S. Freear, "Energy detection based spectrum sensing over $\kappa-\mu$ and $\kappa-\mu$ extreme fading channels," IEEE Trans. Veh. Technol., vol. 62, no. 3, pp. 1031-1040, Mar. 2013.

[38] C. Maccone, "The KLT (Karhunen-Loève transform) to extend SETI searches to broad-band and extremely feeble signals," Acta Astronautica, vol. 67, no. 11-12, pp. 1427 - 1439, 2010.

[39] T. M. Getu, W. Ajib, and R. Jr. Landry, “An eigenvalue-based multi-antenna RFI detection algorithm,” in Proc. IEEE Veh. Technol. Conf. (VTC2018-Fall), Chicago, IL, USA, Aug. 2018, accepted. 
[40] L. L. Scharf and B. Friedlander, "Matched subspace detectors," IEEE Trans. Signal Process., vol. 42, no. 8, pp. 2146-2157, Aug. 1994.

[41] Z. Lu and A. M. Zoubir, "Source enumeration in array processing using a two-step test," IEEE Trans. Signal Process., vol. 63, no. 10, pp. 2718-2727, May 2015.

[42] M. Wax and T. Kailath, "Detection of signals by information theoretic criteria," IEEE Trans. Acoust., Speech, Signal Process., vol. 33, no. 2, pp. 387-392, Apr. 1985.

[43] P. Stoica and Y. Selen, "Model-order selection: a review of information criterion rules," IEEE Signal Process. Mag., vol. 21, no. 4, pp. 36-47, Jul. 2004.

[44] J. G. Proakis and M. Salehi, Digital Communications, 5th ed. New York, NY, USA: McGraw-Hill, 2008.

[45] E. Axell and E. G. Larsson, "A unified framework for GLRT-based spectrum sensing of signals with covariance matrices with known eigenvalue multiplicities," in Proc. IEEE Int. Conf. on Acoust., Speech and Signal Process. (IEEE ICASSP), May 2011, pp. 2956-2959.

[46] G. H. Golub and C. F. Van Loan, Matrix Computations, 4th ed. Baltimore, MD, USA: The Johns Hopkins Univ. Press, 2013.

[47] Q. Huang and P. J. Chung, "An F-test based approach for spectrum sensing in cognitive radio," IEEE Trans. Wireless Commun., vol. 12, no. 8, pp. 4072-4079, Aug. 2013.

[48] A. Taherpour, M. Nasiri-Kenari, and S. Gazor, "Multiple antenna spectrum sensing in cognitive radios," IEEE Trans. Wireless Commun., vol. 9, no. 2, pp. 814-823, Feb. 2010.

[49] P. Wang, J. Fang, N. Han, and H. Li, "Multiantenna-assisted spectrum sensing for cognitive radio," IEEE Trans. Veh. Technol., vol. 59, no. 4, pp. 1791-1800, May 2010.

[50] D. Ramírez, J. Vía, I. Santamaría, and L. L. Scharf, "Detection of spatially correlated Gaussian time series," IEEE Trans. Signal Process., vol. 58, no. 10, pp. 5006-5015, Oct. 2010.

[51] T. M. Getu, W. Ajib, and R. Landry, "Simple F-test based spectrum sensing techniques for multi-antenna cognitive radios," IEEE Trans. Commun., vol. 66, no. 11, pp. 5081-5096, Nov. 2018.

[52] E. Axell, G. Leus, E. G. Larsson, and H. V. Poor, "Spectrum sensing for cognitive radio : State-of-the-art and recent advances,” IEEE Signal Process. Mag., vol. 29, no. 3, pp. 101-116, May 2012.

[53] J. K. Tugnait, “On multiple antenna spectrum sensing under noise variance uncertainty and flat fading," IEEE Trans. Signal Process., vol. 60, no. 4, pp. 1823-1832, Apr. 2012.

[54] L. Scharf and D. Lytle, "Signal detection in Gaussian noise of unknown level: An invariance application," IEEE Trans. Inf. Theory, vol. 17, no. 4, pp. 404-411, Jul. 1971.

[55] D. Ramírez, G. Vazquez-Vilar, R. López-Valcarce, J. Vía, and I. Santamaría, "Detection of rank- $P$ signals in cognitive radio networks with uncalibrated multiple antennas," IEEE Trans. Signal Process., vol. 59, no. 8, pp. 3764-3774, Aug. 2011.

[56] R. R. Nadakuditi and A. Edelman, "Sample eigenvalue based detection of high-dimensional signals in white noise using relatively few samples," IEEE Trans. Signal Process., vol. 56, no. 7, pp. 2625-2638, Jul. 2008.

[57] R. R. Müller, L. Cottatellucci, and M. Vehkaperä, "Blind pilot decontamination," IEEE J. Sel. Topics Signal Process., vol. 8, no. 5, pp. 773-786, Oct. 2014.

[58] H. Yin, L. Cottatellucci, D. Gesbert, R. R. Müller, and G. He, "Robust pilot decontamination based on joint angle and power domain discrimination," IEEE Trans. Signal Process., vol. 64, no. 11, pp. 2990-3003, Jun. 2016.

[59] A. Leshem and A. J. van der Veen, "Multichannel detection of Gaussian signals with uncalibrated receivers," IEEE Signal Process. Lett., vol. 8, no. 4, pp. 120-122, Apr. 2001. 
[60] F. J. Schuurmann, P. R. Krishnaiah, and A. K. Chattopadhyay, "On the distributions of the ratios of the extreme roots to the trace of the Wishart matrix," Journal of Multivariate Analysis, vol. 3, no. 4, pp. 445 - 453, 1973.

[61] P. R. Krishnaiah and F. J. Schuurmann, "On the evaluation of some distributions that arise in simultaneous tests for the equality of the latent roots of the covariance matrix," Journal of Multivariate Analysis, vol. 4, no. 3, pp. 265 - $282,1974$.

[62] A. Maaref and S. Aissa, "Closed-form expressions for the outage and ergodic shannon capacity of MIMO MRC systems," IEEE Trans. Commun., vol. 53, no. 7, pp. 1092-1095, 2005.

[63] A. Zanella, M. Chiani, and M. Z. Win, "On the marginal distribution of the eigenvalues of Wishart matrices," IEEE Trans. Commun., vol. 57, no. 4, pp. 1050-1060, 2009.

[64] F. J. Lopez-Martinez, E. Martos-Naya, J. F. Paris, and A. Goldsmith, "Eigenvalue dynamics of a central Wishart matrix with application to MIMO systems," IEEE Trans. Inf. Theory, vol. 61, no. 5, pp. 2693-2707, 2015.

[65] M. Chiani, M. Z. Win, and A. Zanella, "On the capacity of spatially correlated MIMO Rayleigh-fading channels," IEEE Trans. Inf. Theory, vol. 49, no. 10, 2003.

[66] S. N. Majumdar, "Extreme eigenvalues of Wishart matrices: Application to entangled bipartite system," 2010. [Online]. Available: https://arxiv.org/pdf/1005.4515.pdf

[67] R. Vershynin, High-Dimensional Probability: An Introduction with Applications in Data Science. New York, NY, USA: Cambridge Univ. Press, 2018.

[68] M. J. Wainwright, High-Dimensional Statistics: A Non-Asymptotic Viewpoint. Cambridge, UK: Cambridge Univ. Press, 2019. 